\title{
LES ASPEGTS MÉTROLOGIQUES DES MESURES MAGNÉTIQUES AU SOL ET DANS L'ESPAGE
}

\author{
Par J. PELEN.
}

\begin{abstract}
Résumé. - Les mesures du champ géomagnétique au sol nécessitent la mise en œuvre d'instruments de grande stabilité pour la mesure des variations des composantes du champ (variomètres) ou de grande précision en valeur absolue pour la mesure de l'intensité des modules ou des composantes (magnétomètres).

Les balances magnétiques et les dispositifs à noyaux saturés (fluxgate) peuvent constituer d'excellents variomètres, tandis que seuls les dispositifs à champ total constituent de véritables magnétomètres.

Les mesures du champ géomagnétique dans les couches inférieures de la magnétosphère pour la détermination de l'attitude des engins spatiaux sont effectuées au moyen de magnétomètres triaxiaux et à noyaux saturés (fluxgate).

La mesure des champs magnétiques dans les couches supérieures, ou aux frontières de la magnétosphère, ou dans l'espace interplanétaire, nécessite la mise en œuvre de magnétomètres d'un type nouveau, fonctionnant à champ très faible et avec une très grande sensibilité et une très grande stabilité.
\end{abstract}

1. Après les brillants exposés qui ont rendu compte des remarquables résultats scientifiques obtenus dans plusieurs laboratoires, je voudrais exposer, en l'état actuel de la technique, le point de vue très pragmatique du constructeur, lequel est en contact fréquent avec les utilisateurs, connaît leurs problèmes et leurs exigences.

Devant réaliser des équipements destinés à remplir des missions précises, dans un environnement aux contraintes souvent sévères et dans des conditions opérationnelles souvent difficiles, il doit se conformer à des spécifications techniques rigoureuses.

J'envisagerai dans cet exposé le cas des magnétomètres à composantes et à champ total fonctionnant dans la bande de 0 à $10 \mathrm{~Hz}$, et je formulerai quelques remarques relatives aux difficultés d'ordre métrologique rencontrées dans la mesure des champs magnétiques faibles, qui me paraissent particulièrement aiguës en raison du rapport de plus en plus grand entre l'intensité du champ géomagnétique et la résolution, de plus en plus fine, des magnétomètres.

Je présenterai ensuite succinctement les caractéristiques de quelques magnétomètres construits par la Compagnie des Compteurs.

1.1. Constatons tout d'abord que ces mesures exigent une sensibilité croissante. - $\mathrm{Au}$ sol, certaines études des variations rapides nécessitent d'apprécier le milligamma, en autorisant, il est vrai, des capteurs de grandes dimensions.

Dans l'espace interplanétaire, les mesures sont effectuées aujourd'hui avec un pouvoir de résolution de quelques dixièmes de gamma; mais il serait souhaitable d'apprécier le centième de gamma, avec des appareils de très faible poids et de très faible consommation d'énergie électrique.

1.2. La première remarque concerne les exigences de stabilité. - Un variomètre destiné à un observatoire de géomagnétisme doit apprécier des variations de l'ordre du gamma, à l'intérieur d'une plage de mesure de quelques centaines de gamma, pour une intensité du champ total de l'ordre de $50000 \gamma$; ces conditions exigent de l'appareil un facteur de stabilité à la fois en fonction du temps — sur une période de vingtquatre heures - et des fluctuations de la température ambiante, de l'ordre de $2 \times 10^{-3}$ pour le dispositif de mesure et de $2 \times 10^{-5}$ pour le système de compensation de l'intensité moyenne (courant inducteur ou aimant permanent).

Dans un observatoire terrestre, il est toujours possible de placer le variomètre ou son capteur dans une enceinte à température stable et de procéder à un étalonnage périodique.

La mesure du champ magnétique interplanétaire conduit à un facteur de stabilité beaucoup plus réduit, de l'ordre de $10^{-3}$, mais il importe de conserver cette stabilité au cours d'une très longue période, de l'ordre d'une année, et dans un domaine de température très étendu, pouvant atteindre $100^{\circ} \mathrm{C}$.

Le capteur spatial est disposé à l'extrémité d'un bras, dans des conditions telles qu'il est très difficile d'envisager de le calorifuger ou de le thermostater. En ce qui concerne les possibilités d'étalonnage en vol, la calibration périodique du gain peut être mise en œuvre sans difficulté; il n'en est pas de même de la vérification du zéro des magnétomètres à composantes, laquelle ne peut être obtenue que par un retournement de $180^{\circ}$ du capteur ou par une permutation des axes pour les capteurs triaxiaux.

Un magnétomètre spatial de grande dynamique d'amplitude, qui aurait pour mission d'explorer le champ magnétique à la fois dans les couches inférieures et au-delà de la magnétosphère, suivant une orbite très elliptique, représente le cas extrême, s'il doit pouvoir apprécier le gamma sur toute son étendue de mesure; en effet, il doit posséder un système de compensation stable de $2 \times 10^{-5}$, comme le variomètre d'ob- 
servatoire, mais dans un domaine de température très étendu, de l'ordre de 50 à $100^{\circ} \mathrm{C}$; d'autre part, l'étalonnage en vol à ce niveau de précision est difficilement concevable, si ce n'est avec un magnétomètre à champ total.

1.3. La deuxième remarque concerne le très haut degré de précision absolue souhaité, mais qu'il est rarement possible d'obtenir. - La valeur absolue d'une composante du champ magnétique peut être facilement obtenue au sol à partir du diagramme d'un variomètre stable au moyen d'étalonnages périodiques, si l'on dispose d'une référence absolue.

En fait, il existe peu de références absolues, sûres et de précision suffisante, en dehors des magnétomètres à protons, lesquels délivrent une fréquence de résonance magnétique nucléaire rigoureusement proportionnelle au module du champ magnétique, selon la loi de Larmor.

Mais le magnétomètre à protons ne fournit qu'une seule donnée; pour une détermination complète du vecteur, soit par ses angles d'inclinaison et de déclinaison, soit par ses composantes suivant l'axe vertical et le méridien magnétique du lieu, il est nécessaire de recourir à un système de bobines convenablement orientées, permettant de composer vectoriellement un champ magnétique auxiliaire avec le champ géomagnétique, par exemple suivant la méthode d'Alldredge, qui permet une détermination directe des valeurs numériques des angles d'inclinaison et de déclinaison.

En ce qui concerne les mesures dans l'espace, l'emploi des magnétomètres à champ total est resté limité jusqu'ici, car peu d'entre eux sont capables de fonctionner pour des intensités très faibles de l'ordre de quelques gammas.

Néanmoins, le magnétomètre à hélium a été utilisé sur certaines sondes Mariner, comme nous l'a exposé M. Slocum, pour la détermination des trois composantes du champ interplanétaire et le magnétomètre à vapeur de rubidium sur le satellite Ogo 5, comme nous l'a exposé M. Ledley. Dans la plupart des cas, il est fait appel aux magnétomètres à fluxgate triaxiaux.

Jusqu'à présent, ces appareils sont considérés comme de bons variomètres; les récents progrès obtenus par quelques constructeurs permettent d'obtenir une excellente stabilité à long terme, dans un domaine de température de l'ordre de $80^{\circ} \mathrm{C}$, ainsi qu'un bruit de fond de $0,2 \gamma$ crête à crête dans la bande de 0 à $10 \mathrm{~Hz}$, de sorte qu'il est possible d'espérer une dérive globale inférieure au gamma lorsque l'intensité n'excède pas quelques dizaines de gammas.

Le but recherché étant la mesure de la valeur absolue des composantes, de manière à restituer le vecteur en module et angles, il est nécessaire de procéder à une rigoureuse définition de l'axe magnétique et du zéro magnétique des magnétomètres à fluxgate.

1.4. Notre troisième remarque concerne la difficulté de s'affranchir des perturbations magnétiques lors des étalonnages, et plus particulièrement d'obtenir un champ rigoureusement nul; il en a été beaucoup question lors de ce colloque et d'excellentes solutions ont été proposées.

Bien entendu, on recherche tout d'abord un site calme et propre, où les perturbations industrielles ne se fassent pas ressentir, où le gradient de champ naturel soit aussi faible que possible.

Il est relativement facile de s'affranchir des varia- tions naturelles, soit en les filtrant par un blindage magnétique, soit en les compensant avec des bobines. Pour effectuer un étalonnage en champ nul, on utilise une série de blindages en permalloy ou en mu-métal, de forme sphérique ou cylindrique; les variations du champ magnétique sont atténuées très efficacement, jusqu'à $10^{-5}$ et même $10^{-6}$; mais du fait de la rémanence du matériau, il subsiste un champ résiduel de plusieurs dizaines de gammas, qui peut être réduit par désaimantation.

Il est éventuellement possible de compenser ce champ résiduel à l'aide d'un petit bobinage intérieur au blindage.

Il est assez commode de procéder par retournement de capteur; en opérant avec une précision angulaire suffisante, on peut ainsi s'affranchir du champ résiduel.

Les blindages à supraconducteur qui ont été décrits lors de ce colloque permettront certainement, lorsqu'ils seront disponibles sur le marché, d'améliorer considérablement la définition du zéro des magnétomètres.

1.5. Notre dernière remarque concerne la contamination magnétique par le véhicule portant le magnétomètre. M. Vuye nous a exposé les moyens assez considérables mis en œuvre pour assurer la propreté magnétique du satellite Heos A1, et M. Iufer nous a décrit les installations dont dispose le Ames Center de la Nasa.

Généralement, le magnétomètre est situé à l'extrémité d'un bras amagnétique, dont la longueur, pour des raisons de rigidité, n'excède guère 2 à $2,5 \mathrm{~m}$.

La structure et les composants de l'engin spatial sont sélectionnés en fonction de leur amagnétisme et sont soumis au cours des différentes phases de fabrication à une série de traitements de décontamination et de tests de contrôle magnétique; les circuits électriques parcourus par des courants continus sont disposés de manière à minimiser les champs de fuite. Malgré toutes ces précautions, il est difficile d'obtenir un niveau de perturbations inférieur à quelques dixièmes de gamma, qui est susceptible d'être modifié par magnétisation lors des opérations de lancement.

Pour cette raison, il paraîtrait illusoire, dans l'état actuel des choses, de mettre en œuvre sur des engins spatiaux des magnétomètres dont la résolution serait inférieure au dixième de gamma, à moins de pouvoir disposer d'une méthode de compensation automatique adaptée et de pouvoir la mettre en œuvre efficacement sur le véhicule spatial.

2. Pour illustrer cet exposé, nous présenterons succinctement trois magnétomètres construits par la Compagnie des Compteurs.

2.1. Tout d'abord un variomètre à fluxgate type HAS 911 pour mesures analogiques au sol, dont la résolution est de $0,5 \gamma$, le bruit de fond de $0,2 \gamma$ crête à crête, la stabilité à long terme de $0,5 \gamma$ par semaine à température constante, la stabilité en fonction de la température de $0,02 \gamma$ par ${ }^{\circ} \mathrm{C}$.

L'appareil comporte un dispositif de compensation pour effectuer des mesures entre -100000 et $+100000 \gamma$ dont la précision propre est de $10^{-4}$ et dont la stabilité en fonction de la température de $0,5 \times 10^{-6}$ par $^{\circ} \mathrm{C}$. Le changement de plage de réglage est effectué automatiquement au moyen d'un dispositif de stepping à 18 pas, qui permet de couvrir sans intervention une étendue de mesure totale de $2000 \gamma$.

L'axe magnétique du capteur est défini à mieux 
que $0,1^{\circ}$ et le zéro magnétique à mieux que $0,2 \gamma$. La bande passante est de $16 \mathrm{~Hz}$ à $3 \mathrm{~dB}$. L'effet de mémoire magnétique pour un champ inducteur de 5 Oe n'est pas perceptible dans le bruit de fond.

Un appareil de ce type a été fourni au champ de tir de l'Esro à Kiruna pour la prévision et l'étude des aurores boréales. Cet appareil est susceptible d'être transposé en version spatiale.

2.2. En second lieu, un magnétomètre d'attitude construit pour le satellite Esro 1 (Aurorae) lancé le 3 octobre 1968. - Comportant quatre capteurs élémentaires avec une étendue de mesure de -60000 à $+60000 \gamma$ pour les deux premiers et de -6000 à $+6000 \gamma$ pour les autres, cet appareil est utilisé pour la restitution d'attitude du satellite, lequel est stabilisé suivant les lignes de force du champ géomagnétique.

Suivant l'objectif assigné, les angles de dépointage doivent être déterminés avec une précision de l'ordre du degré; il n'était donc pas nécessaire de rechercher une grande résolution; l'effort a porté sur la simplification des circuits en vue d'accroître la fiabilité et sur la réduction du poids (inférieur à $400 \mathrm{~g}$ pour l'ensemble électronique et capteurs relatifs à deux axes) et de la consommation électrique (de l'ordre de $150 \mathrm{~mW}$ pour deux axes). Les performances à signaler particulièrement sont la linéarité, de l'ordre de 0,5 pour mille, et la stabilité en fonction de la température, qui est telle que l'appareil conserve sa précision entre - 20 et $+70^{\circ} \mathrm{G}$.

Sur le même principe ont été réalisés les magnétomètres du satellite français D2A; ceux du satellite français Eole sont en cours de réalisation et présentent des performances améliorées, notamment au point de vue de la consommation qui est réduite à $30 \mathrm{~mW}$ pour trois axes.

Sur le même principe également sont construits les magnétomètres triaxiaux pour fusée-sonde, type HRA 31, qui admettent des tensions d'alimentation comprises entre 10 et $40 \mathrm{~V}$ et permettent les modes de sortie compatibles avec les différents systèmes de télémesure.

Ces magnétomètres, qui équipent depuis plusieurs années les fusées-sondes des programmes français et européens, sont particulièrement remarquables par leur faible encombrement, leur faible poids $(600 \mathrm{~g}$ pour 3 axes) et leur faible consommation d'énergie électrique ( $250 \mathrm{~mW}$ pour 3 axes).

2.3. Un magnétomètre à champ total FP1 $A$, pour les mesures absolues des champs magnétiques au sol avec une grande précision en valeur absolue; construit sous licence G.E.A., cet appareil utilise le principe de la résonance magnétique nucléaire avec polarisation dynamique par effet Overhauser-Abragam et avec entretien du signal nucléaire, qui a été décrit par M. Salvi.

Comme M. Miquel l'a exposé, cet appareil permet une mesure continue du champ magnétique et comporte une sortie analogique sur enregistreur graphique, avec une résolution de $0,01 \gamma$ et une sortie digitale comportant un affichage numérique, avec une résolution de $0,1 \gamma$.

Sa précision en valeur absolue est de $1 \gamma$; le capteur qui présente une erreur d'anisotropie inférieure à $0,1 \gamma$ peut fonctionner sans aucune erreur entre - 50 et $+70^{\circ} \mathrm{C}$ et ne nécessite aucun entretien.

Le même appareil existe en version simplifiée portable type FP 25 avec sortie digitale et affichage numérique incorporé, présentant une résolution de $0,25 \gamma$ et une précision de $1 \gamma$.

Une autre version en cours de développement constituera l'élément essentiel d'un observatoire automatique permettant de mesurer, avec toute la précision absolue désirable $(1 \gamma)$, les valeurs numériques du module du vecteur et des angles d'inclinaison et de déclinaison.

Ces différents magnétomètres peuvent également être réalisés en version différentielle, soit à sortie analogique, soit à sortie numérique. 